\title{
The influence of two rehabilitation protocols in upper limb function of stroke patients
}

\section{Marlene Rosa Oloa Vasconcelos Alda Marounes}

Abstract:

A significant decrease of upper limb functionality occurs after stroke (Nunes et al., 2005). There are two different approaches on upper limb rehabilitation of stroke patients: unilateral and bilateral protocols (Byblow and Lewis, 2004). The main goal of this study was to assess the differences in the functionality of the hemiparetic upper limb (HUL) among individuals with chronic hemiparesis submitted to a Unilateral Protocol (UP) and those submitted to a Bilateral Protocol (BP). Eight individuals with stroke and chronic hemiparesis were recruited and randomly divided in two groups (four participants in each group). All participants were followed during six weeks and evaluated at four different moments of the intervention protocol using the Fulg-Meyer (FM) Assessment Scale (Fulg Meyer et al. 1975) and the Purdue Pegboard (PP) (Tiffin, 1948). The statistical procedures included percentage evolution and descriptive analysis for each participant. The findings suggest that the UP presented more favorably evolution, considering the FM $(9 \%, 15 \%, 15 \%, 0 \%)$ and the PP $(18 \%, 8 \%,--, 10 \%)$, when compared with the BP for the FM $(0 \%,-1 \%, 11 \%,-22 \%)$ and PP $(1 \%, 19 \%,--$, $0 \%$ ).

Therefore, this study suggests that Unilateral Protocols are at least as effective as Bilateral Protocols on hemiparetic upper limb rehabilitation.

Keywords: stroke, hemiparetic upper limb, function rehabilitation, unilateral protocol, bilateral protocol.

\section{Introduction}

Stroke is the second cause of death (Silva, 2004) and the first cause of morbidity and incapacity worldwide (Kulkens and Rigleb, 2003). The most important clinical characteristics of stroke patients are motor and sensitive impairments, with significant decrease of physical function (Samela et al., 1999). The upper limb disability represents a significant impairment for these patients (Feys et al., 1998) and its recovery has been considered to be challenging (Byblow and Lewis, 2004). The main protocols for upper limb rehabilitation can be classified as unilateral and bilateral. The recent literature has distinguished the Bilateral Arm Training with Rhythmic Auditory Cueing (BATRAC) due to its positive results showed in global motor coordination specifically, in upper limb coordination and range of movement (Sandy et al., 2004). The BATRAC consists of a platform with two independent bars in a form of " $T$ " which moves in a transverse plan, perpendicular to the patient. These bars create upper limb movements (see Figure 1), promoting the flexion of the shoulders and extension of the elbows, and extension of the shoulders and flexion of the elbows, on "rhythm" and "repetitive" movements - two important concepts on upper limb rehabilitation defended by Whitall et al. (2000). 


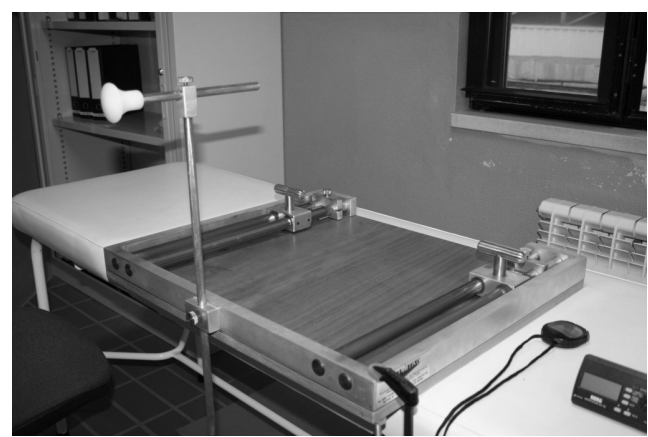

Figure 1: Repetitive Bilateral Arm Training With Rhythm Auditory Cueing (BATRAC) platform.

Research evidence of the effects of these two approaches (unilateral and bilateral) remains controversial and the different aspects that may influence neurological rehabilitation, especially on not-acute phase it is still unknown. Therefore, the aim of this study was to investigate patients with chronic stroke and to describe their responses to the two upper limb rehabilitation protocols.

\section{Literature review}

Stroke contributes to 5 million deaths and more than 15 million survivors per year worldwide (Silva, 2004) and in Europe the mortality varies between 63,5 and $273,4 / 100000$.

Stroke it is also an important cause of disability which is related to a range of factors such as: area and extension of the injury, muscle tonus and stereotype movements (Samela et al., 1999), gender, age, affected side, time after stroke, quality of the rehabilitation, patient motivation, family follow-up (Sullivan and Schimtz, 2000) and the integrity of the sensory system (Teixeira et al., 1999). All these factors may impact on the ability to carry out activities as walking, climbing stairs or self-caring and therefore, must be considered in the functional recovery of neurological patients (Sullivan and Schimtz, 2000, Formisano et al., 2005 and Teixeira et al., 1999). Furthermore, more than $85 \%$ of the patients show a deficit of the hemiparetic upper limb (Feys et al., 1998). The recovery process of the upper limb includes a complex reeducation of muscular activity of the shoulder and fingers, which is of main importance to many daily activities e.g., to grab or manipulate objects (Feys et al., 1998). The complications of upper limb recovery (e.g., shoulder subluxation, shoulder-hand syndrome, injuries of soft tissues and shoulder pain) and the lack of spontaneous stimulation (as many daily tasks are performed using only the non-affected upper limb) might explain the remain deficits of this extremity on the initial rehabilitation period (Feys et al., 1998). Therefore, research on upper limb rehabilitation needs to be conducted as spontaneous recovery is potentially weak (Feys et al., 1998). Physiotherapy treatments are often part of the upper limb rehabilitation, and in general, unilateral and bilateral protocols are applied however, the research evidence about them is controversial (Winstein et al., 2004). Byblow and Lewis (2004) suggest that lack of cortical representation of the hemiparetic upper limb should be compensated by ipsilateral stimulation, and therefore these authors defend the use of unilateral interventions. However, Cauraugh and Kim (2002) defends that bilateral interventions are more effective in the improvement of the voluntary motor control and Carson et al. (2005) claim neurophysiologic evidence that supports the bilateral intervention. The latter authors defend a systematic and natural interaction in the upper limbs and believe that the functional improvement of the hemiparetic muscles is obtained more easily through bilateral movements. Hortobagyi et al. (2003), also followed this principle and 
investigated the movements of the contralateral muscles, when isometric contractions on wrist flexors were requested. It was suggested that a unilateral voluntary muscle contraction causes contralateral effects at both cortical and segmental levels. Similarly, investigating six stroke patients during a rehabilitation protocol using a cycle ergometer with the two upper limbs Godoi et al., 2007, concluded that this protocol caused benefits to the paretic upper limb. Both approaches (unilateral and bilateral) seem to be beneficial to the hemiparetic upper limb function recovery however, more research is needed as no consensus is yet found. Therefore, this study aimed to verify the effect of two rehabilitation protocols (unilateral and bilateral) and to understand its impact on the functionality of the hemiparetic upper limb.

\section{Methods}

\subsection{Methodology}

This was a series of eight case studies of stroke patients submitted to different upper limb rehabilitation protocols. All patients provided informed consent prior to data collection and were randomly divided in two groups (4 participants each). One group was submitted to a unilateral rehabilitation protocol and the second group to a bilateral rehabilitation protocol. A purposive sampling was used based on the inclusion criteria described below, and on the nearest geographical area. Ethical approval was given by the health institutions involved.

\subsection{Setting, Interventions and Timescales}

A purposive sample of eight stroke participants with chronic hemiparesis - with, at least, six months after stroke (Stinear et al., 2007) - were recruited from two hospitals (Anadia and Águeda) and private institutions (clinics). A specific questionnaire based on Whitall et al. (2000) was applied to ensure that the inclusion criteria were accomplished, i. e., antigravitational movement of the hemiparetic shoulder, minimal of six weeks after stroke and Mini Mental State Examination $\geq 22$ points.

Each participant group was followed during six weeks (standard rehabilitation period). Data were collected in four time points at: T1 (baseline), T2 (2 weeks post intervention), T3 (4 weeks) and T4 (last treatment) of the intervention. The research began in January 2007 with the Bilateral Group intervention and was followed by the Unilateral Group. The research study finished by April 2007.

\subsection{Outcome measures}

Demographic and clinical data was first collected. To measure the motor and the sensorial commitment of the hemiparetic upper limb, the Fulg-Meyer Evaluation Scale was applied (Cacho et al., 2004). In this scale the final score is a cumulative numerical score of: motor recovery, balance, sensation, pain and joint range of movement. Each item is scored on a three point scale: $0=$ can not perform, $1=$ partially perform, $2=$ fully perform. The FM scale is highly recommended as a clinical and research tool for evaluating changes in motor impairment following stroke (Gladstone et al., 2002). The Purdue Pegboard created by Tiffin (1948), following specific rules defended by Hebert et al., 1995 was then used to score manual dexterity. This is an instrument to select and rehabilitate manual dexterity of the fingers, hands and upper limb movements (simple and associated ones). The reliability of the Purdue Pegboard test is high and it has been widely used to test dexterity in clinical settings with in stroke and multiple sclerosis patients (Crafton et al., 2003). It consist of a frame with pins, circles, cylinders and 
some specific tests where all pieces should be placed on the frame by the left, right and both hands, depending of selected test ("right hand"; "left hand"; "both hands" or "assembly" tests).

All measures were applied following the described order to all participants at the four time points (T1, T2, T3, T4).

\subsection{Interventions}

The two groups of participants were submitted to the respective protocol (Unilateral and Bilateral) during six weeks, three times a week.

The group submitted to the bilateral protocol used the BATRAC platform (repetitive bilateral arm training with rhythmic auditory cueing) and performed the intervention recommended by Whitall et al. (2000). In each session, each individual was submitted to four intervention periods, five minutes duration each, and the participant had to achieve the platform with both upper limbs. Participants rested for ten minutes between each period of exercises. Cardiac frequency and blood pressure were measured at beginning and at the end of each set to assess if it would be necessary to increase the period of rest, until hemodynamic stabilization. The movement performed with the platform bars was to bring up and push the two bars in " $T$ " using the noise of a stop watch to time the trainings. This movement is composed by shoulder abduction and flexion/extension elbow, which represent the functional upper limb movement to reach an object.

The comfortable rhythm for each patient to perform the tasks was chosen in the first session and remained constant throughout the study.

The group submitted to the unilateral protocol used the same platform and also the Manual Dexterity Test of Minnesota (MDTM) to equal the "active time" of the intervention between both groups. The MTDM were added to the unilateral group because during a pilot study it was found that whilst the participants under the bilateral group would move the BATRAC bars e.g. 20 times without major difficulties, the unilateral group would move just a few times and get tired. This resulted on a shorter "active time" of the intervention in the unilateral group. As the BATRAC protocol is very well defined, the only possible way to equal the "active time" of the intervention was to add a new set of tests. The Minnesota Tests has been chosen because they are found to be a valid assessment and training tool for upper limb stroke rehabilitation (Lourenção et al, 2006). Therefore, despite of the different time of sessions in each group, using this methodological approach, the opportunity of active participation in both groups became similar. Therefore, despite of commonly the MTDM being used as an outcome measure, in this study its protocols were used as part of the unilateral intervention.

BATRAC was used following the same procedures as the previous group however, the bars were moved using exclusively the hemiparetic upper limb. The MDTM is a board with 60 holes and a set of 60 round pieces which measures and evaluates the global motor capacities of the upper limb. It was adapted to hemiparetic patients by Lourenção et al., (2006). This test was applied through the "Position Test" "and "Return Test" during ten minutes each. For the Position Test, it is necessary that the patient places all the round pieces in the board only with the hemiparetic hand, starting from the column of the ipsilateral side to the affected one, from top to bottom. After filling a column, the patient can fill the next one, until he/she completed all board. For the Return Test, the patient puts back the round pieces with the hemiparetic hand, turning around the face of each one. During this replacement, the patient executes an "S" line, i.e., first, completes the top line, starting from his/her affected side, and then fills the line below from the side where he/she finished the previous one. 


\section{ระ:

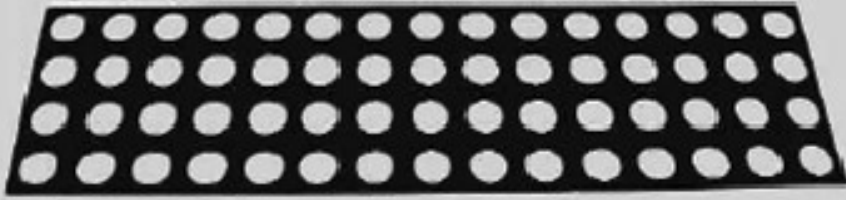

Figure 2: Manual Dexterity Test of Minnesota (MDTM) instrument.

\subsection{Statistical Analysis}

All data was introduced into SPSS version 14,0. Data was organised first per patient at each evaluation moment of the study and then from all patients globally to look for evolution patterns. The analysis consisted of descriptive analysis (mean and standard deviation) and percentage of evolution (from T1 to T4). The percentage of evolution was calculated using the equations (1) and (2) (Antunes, 2004).

$\left[\left(\mathrm{FM}_{\mathrm{T} 4}\right)-\left(\mathrm{FM}_{\mathrm{T} 1}\right) /\left(\mathrm{FM}_{\mathrm{T} 4}\right)\right] \times 100$

Equation 1: Percentage of evolution of Fulg-Meyer sensory motor scale (FM) scores

$\left[\left(\mathrm{PP}_{4} 4\right)-\left(\mathrm{PP}_{11}\right) /\left(\mathrm{PP}_{\mathrm{T}}\right)\right] \times 100$

Equation 2: Percentage evolution of Purdue Pegboard Test (PP) scores

\section{Findings}

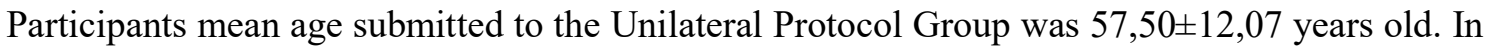
the Bilateral Protocol Group participants mean age was 54,75 $\pm 5,50$ years old. Table 1 characterise the sample in each intervention protocol, in terms of age (years), gender ( $\mathrm{M}=$ male and $\mathrm{F}=$ female) and time after stroke (months).

\begin{tabular}{lllllllll}
\hline & \multicolumn{3}{l}{ Unilateral Protocol Group } & \multicolumn{4}{c}{ Bilateral Protocol Group } \\
\hline Participants & H & ME & M & R & C & J & L & MA \\
\hline Age (years) & 65 & 66 & 59 & 40 & 51 & 56 & 63 & 50 \\
\hline Gender (F/M) & M & F & M & F & M & M & F & F \\
\hline Time after stroke (months) & 72 & 9 & 27 & 22 & 19 & 49 & 22 & 131 \\
\hline
\end{tabular}

Table 1: Characteristics of the participants under the Unilateral (UP) Protocol Group and the Bilateral (BP) Protocol Group.

The Fulg-Meyer Scale results are presented in Graph 1 for the Unilateral Protocol Group and in Graph 2 for the Bilateral Protocol Group.

In the Unilateral Protocol Group, there were three (H., ME. and R.) of the four participants with a similar evolution throughout the study. The fourth participant (M.) presented worse scores and an irregular pathway in its evolution, ending the intervention protocol with a slightly higher value than at baseline.

In the Bilateral Protocol Group, there were also two participants (C. and L.) with a similar evolution throughout evaluation moments. One of these two participants maintained its scores stable from T1 to T4 (C.) and the second participant (J.) showed a decreased in T2 but recovered to the baseline value in T3 and T4. The other two participants (L. and MA.) presented lower scores $([5 ; 20])$. It is important to note that participant J. showed good evolution of its performance, when comparing $\mathrm{T} 1$ to $\mathrm{T} 4$ however, throughout the study, its score decreased (T3), which showed that its pattern of evolution was not linear during the intervention period. Participant MA. ended the study with a lower score than at baseline, presenting a negative percentage of evolution, as it can also be observed in table 2 . 

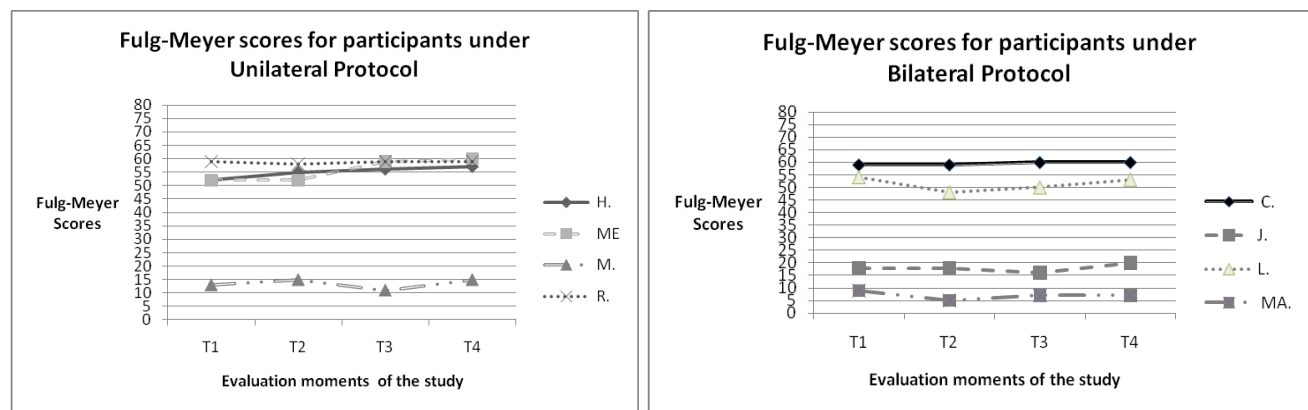

Graph 1 and Graph 2: Evolution curves of the Fulg-Meyer Scores through the evaluation moments (from T1 to T4) for the participants under the Unilateral and the Bilateral Protocol Groups, respectively.

The Purdue Pegboard absolute values since T1 to T4 may be observed in Graph 3 for the Unilateral Group and in Graph 4 for the Bilateral Group.

Three participants under the Unilateral Group showed improvements in this test (R, ME. and $\mathrm{H}$ ) whereas in the bilateral group only one participant (L.) obtained at the end of the study (T4) a higher result than at baseline (T1). In the Unilateral Protocol Group, two participants (R. and ME.) presented a range of scores higher $([60 ; 75])$, than the other participants under the same intervention and also than the Bilateral Protocol Group.
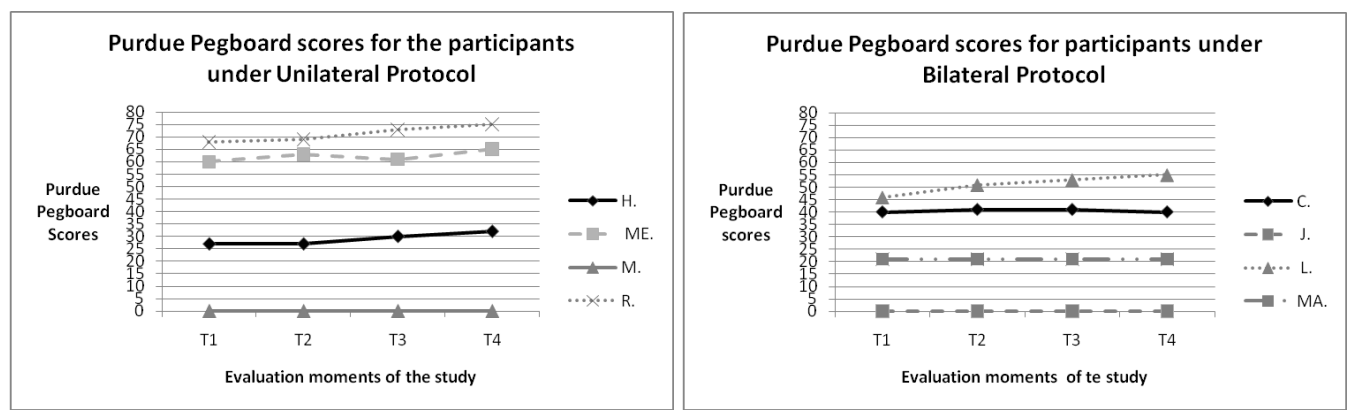

Graph 3 and Graph 4: Evolution curves of Purdue Pegboard Scores through the evaluation moments (from T1 to T4) for the participants under the Unilateral and Bilateral Protocol Groups, respectively.

Table 2 presents the percentage of evolution during the study in both scales. In the Unilateral Group the percentage of evolution was positive in all participants, with the exception of participant R. (who maintained its score stable in the Fulg Meyer scale from T1 to T4). The absence of values for the patients J. and M. in Purdue Pegboard Test, means that these individuals were classified "not suitable" (NS) for this instrument as they were unable to handle the Purdue Pegboard pieces.

In the Bilateral Group two participants presented negative percentage of evolution (L. and MA.), both in the Fulg-Meyer scale and participant MA. did not improve in the Purdue Pegboard test either. The percentage of evolution on the Fulg-Meyer scale was found to be positive in participant J. however participant C. did not improve on the Fulg-Meyer scale and its percentage of evolution on the Purdue Pegboard was lower.

Therefore, overall the Unilateral Group presented a more favorably percentage of evolution measured by these two outcome measures, concerning participants H., ME. and M. for the FulgMeyer scale and participants H., ME. and R. for the Purdue PegboardTest, without negative percentage of evolution values. 


\begin{tabular}{ccc}
\hline Participants & $\begin{array}{c}\text { Percentage evolution } \\
\text { Fulg-Meyer }\end{array}$ & $\begin{array}{c}\text { Percentage evolution } \\
\text { Purdue Pegboard }\end{array}$ \\
\hline Unilateral Protocol Group & & $18 \%$ \\
\hline H. & $9 \%$ & $8 \%$ \\
\hline ME. & $15 \%$ & $\mathrm{NS}$ \\
\hline M. & $15 \%$ & $10 \%$ \\
\hline R. & $0 \%$ & $1 \%$ \\
\hline Bilateral Protocol Group & & $19 \%$ \\
\hline C. & $0 \%$ & $\mathrm{NS}$ \\
\hline L. & $-1 \%$ & $0 \%$ \\
\hline J. & $11 \%$ & \\
\hline MA. & $-22 \%$ & \\
\hline
\end{tabular}

Table 2 - Participants' percentage of evolution for the Fulg Meyer scale and for the Purdue Pegboard test .

\section{Discussion}

The findings suggest that the Unilateral Group presented more favorably evolution, considering the FM Scale $(9 \%, 15 \%, 15 \%, 0 \%)$ and the PP test $(18 \%, 8 \%$, NS, $10 \%)$, when compared with the Bilateral Group for the FM scale $(0 \%,-1 \%, 11 \%,-22 \%)$ and PP test $(1 \%, 19 \%, \mathrm{NS}, 0 \%)$. These results are in conflict with the studies developed by Carson et al. (2005), Cauraugh and Kim (2002) and Hortobagyi et al. (2003), as it is defended that more positive results of the hemiparetic upper limb rehabilitation are obtained, when patients are submitted to bilateral protocols however, in accordance with Byblow and Lewis (2004) conclusions, which defend the unilateral protocols as the most effective interventions for the upper limb recovery.

Despite these findings, it is important to mention that patient's baseline measures were very different, so it is difficult to exclude the idea that the occurred change was a result of a pattern of variability of each participant. This was an important limitation of this study, which should be taken into account in future research. Therefore, this study suggests that Unilateral Protocol was at least as effective as Bilateral Protocol but remains unresolved the lack of consensus of the literature and also the difficulty of health professionals in choosing the most adequate intervention for stroke patients who need upper limb rehabilitation. It can therefore be concluded that more research is needed to increase the knowledge in this area and develop clinical evidence-base practice.

In this research study, globally the manual dexterity (measured in PP test) presented more positive results than the sensorial-motor function evolution (measured in FM scale). It is the authors' opinion that this should be further investigated to understand if this has been an occasional finding or if manual dexterity tends to better recover than sensorial-motor function. A study like this would have clinical implications and contribute for the development of the rehabilitation clinical practice.

The small sample size of this study can be considered a limitation of this study however, only following this methodology has been possible to have the same physiotherapist to follow the participants under both interventions during a longer protocol period (six weeks). This represents a simultaneously methodological disadvantage and advantage. Therefore, ideally it is recommended to develop further research studies with a larger sample and control the effects which can be generated by the fact of having different physiotherapists involved in the interventions. The lack of blinding of the assessor and treatment provider can also be considered another limitation of this study. However, as this was a research study in a clinical setting the blinding is very difficult to address. In future research this should consider and also a patients follow-up, to promote knowledge of the long-term effects of these protocols.

Finally, the BATRAC protocol has been created for bilateral interventions however, it was used in this study because it uses rhythm and repetitive action during the motor activity which is considered to have success in upper limb recovery (Whitall et al., 2000). Therefore, in the future it would be interesting to develop the BATRAC "concept" applied to unilateral interventions and consequently not to have different "active time" of the interventions in both groups. This 
would allow stronger conclusions to be taken regarding upper limb rehabilitation protocols and influence clinical decisions.

\section{Conclusion}

This study suggests that Unilateral Protocol was at least as effective as Bilateral Protocol however, the lack of consensus of the literature about the effects of these protocols on hemiparetic upper limb rehabilitation remains unresolved. Therefore further research is recommended, to optimise patients' upper limb recovery, help health professionals on their clinical practice decisions and increase evidence-base practice on upper limb rehabilitation.

\section{References}

1. Bagg, S., Pombo, P., Hopman, W. Effect of age on functional outcomes after stroke rehabilitation. American Heart Association. 2002; 33-179.

2. Blackburn, M., Paulette, V., Simon M. Reliability of Measurements Obtained With the Modified Ashworth Scale in the Lower Extremities of People With Stroke. PHYS THER. 2002. Vol. 82, pp. 25-34.

3. Byblow, WD., Lewis GN. Neurophysiological and behavioral adaptations to a bilateral training intervention in individuals following stroke. Clin Rehabil. 2004. 18:48-59.

4. Cacho, A., Lins F., Melo, R., Oliveira, R. Avaliação da recuperação motora de pacientes hemiplégicos através do protocolo de desempenho físico Fulg-Meyer. 2004. Revista de Neurociências. Vol.12, n2.

5. Cambier, D., Danneel, A., Witvrouw E. Treating sensory impairments in the post-stroke upper limb with intermittent pneumatic compression. Results of a preliminary trial. Clinical Rehabilitation. 2003. Vol. 17, No. 1, 14-20.

6. Carson, R. Bilateral interactions between the upper limbs. Physiology News. 2005; (58): $37-38$.

7. Cauraugh, H., Kim, S.. Two coupled motor recovery protocols are better than one - Electromyogram-triggered neuromuscular stimulation and bilateral movements. Stroke.2002; 33: 1589 - 1594.

8. Crafton, K., Mark, A., Cramer, S. "Improved understanding of cortical injury by incorporating measures of functional anatomy “.Brain, Vol. 126, No. 7, 1650-1659, July 2003.

9. Desrosiers J., Rochette, A., Hebert, R., Bravo, G.. The Minnesota Manual Dexterity Test: Reliability, Validity and Reference Values Studies with Healthy Elderly People. Canadian Journal of Occupational Therapy.1997. 64: 270-76.

10. Desrosiers, J., HŽbert, R., Bravo, G. Dutil, $f$. The Purdue Pegboard Test: normative data for people aged 60 and over. Disabil. Rehabil.1995. 17: 217-224

11. Feys, M., Weerdt, J., Selz, E., Steck, C., Spichiger, R., Vereeck, E., Putman, D., Hoydonck, V. Effect of a therapeutic intervention for the hemiplegic upper limb in the acute phase after stroke - A Single-Blind, Randomized, controlled multicenter trial. Stroke. 1998. 29: $785-792$.

12. Fess, E. Guidelines for evaluating assessment instruments. Journal of Hanf Therapy, 8, 144-148.

13. Formisano, R., Pantano, P., Buzzi, G., , Vincenzo, P., Francesca, B., Piero, L., Gian L. Late motor recovery is influenced by muscle tone changes after stroke. Archives of Psysical Medicine and Rehabilitation.2005. 86(2):308-11.

14. Gladstone, D., Danells, C., Black, S. "The Fugl-Meyer Assessment of Motor Recovery after Stroke: A Critical Review of Its Measurement Properties". Neurorehabilitation and Neural Repair, Vol. 16, No. 3, 232-240 (2002).

15. Godoi, J., Bagesteiro, L.B., Alouche, S.R. Electromyographic analysis of stroke patients affected-limb while cycling on an arm ergometer. 2007. Motor Control (11): p. S226-S226.

16. Gray, L. ;Sprigg, N.; Bath,W.; Boysen, G.; Deyn, P.P.; Leys, D.; O’Neill, D.; Ringelstein, E.B. Sex differences in quality of life in stroke survivors: data from the tinzaparin in acute ischaemic stroke trial (TAIST). Stroke. 2007. 38(11): $2960-2964$.

17. Haase, V.G, Lacerda, S.S. Neuroplasticidade, variação interindividual e recuperação funcional em neuropsicologia. Temas em Psicologia da SBP. 2004. 17, 28-42.

18. Hamilton, B., Heinemann, A., Linacre, J., Wright, B., Granger, C. Prediction of rehabilitation outcomes with disability measures. Archives of Physical Medicine and Rehabilitation. 1994. 75(2), 133-143.

19. Henriques, D. Paraplegia - Percursos de adaptação e qualidade de vida. Edit Formasau, 2004. 1st. Edition.

20. Hortobagyi, T., Taylor, J., Peterson, N. Changes in segmental and motor cortical output with contralateral muscle contractions and altered sensory inputs in humans. Neurophysiology. 2003. 90: 2451-2459.

21. Jocelyn E., Harris, O. Individuals with the Dominant Hand Affected following Stroke Demonstrate Less Impairment Than Those with the Nondominant Hand Affected. Neurorehabilitation and Neural Repair. 2006. Vol. 20, No. 3, 380-389.

22. Katoh, J., Ohsaka, G., Hara, Y., Ishihara, K., Taniguchi, H., Narutaki, K. "ADL Assessment for Hemiparetic Stroke Patients Based on the Functional Independence Measure". Journal of Physical Therapy Science. Vol. 8 (1996), No. 1 9-12.

23. Kulkens, S., Ringleb, P. AVC isquémico: profilaxia e tratamento. European Stroke Initiative. 2003.

24. Lourenção, M., Tsukimoto, G. Battistela, L. O teste de destreza manual de Minnesota adaptado "utilizado como avaliação do potencial de uso de membros superiores de pacientes hemiplégicos. (2006). 14. 56-61.

25.. Moura, W.; Campo S., Priscilla A. Fisioterapia - Aspectos clínicos e práticos da reabilitação. São Paulo, 2005. Edit by Artes Médicas, Lda.

26. Nancy, M., Bonifer A., Kristin A. Application of Constraint-Induced Movement Therapy for an Individual With Severe Chronic Upper-Extremity Hemiplegia. Phys. Ther. 2003. 83. 4. 384-398.

27. Nunes, S.; Pereira, C.; Gomes S. Evolução funcional de utentes após AVC nos primeiros seis meses após a lesão. Essfisionline. 2005. 1(3): $3-20$

28. Paolucci, S., Bragoni, M., Coiro, P., Angelis, D., Fusco, R., Morelli, D., Venturiero, V., Pratesi, L. Is sex a prognostic factor in stroke rehabilitation?. Stroke. 2006. 37: 2989 - 2994.

29. Popescu, S.; Poenaru D.; Cinteza, D.; Ionita, L.; Mateescu M. Shoulder Pain Management in stroke. Maedica A Journal of Clinical Medicine, 2008. Vol.3.

30. Salmela, T.; Olney, .J.; Nadeau, S.; Brouwer, B. Muscle strengthening and physical conditioning to reduce impairment and disability in chronic stroke survivors. Arch. Phys. Med. Rehabil. 2000. 80.1211-1218.

31. Sandy W., Jill W., Larry F., Richard M., John S., Jorg S., Andrew G., Daniel H. Repetitive Bilateral Arm Training and Motor Cortex Activation in Chronic Stroke. A Randomized Controlled Trial. JAMA.2004;292:1853-1861.

32. Silva, F. Acidente vascular cerebral isquémico - Prevenção: Aspectos actuais - é preciso agir. Medicina Interna. 
2004. 11 (2): 99 - 106.Sullivan, S., Schmitz, T. Fisioterapia: avaliação e tratamento. $3^{\text {a }}$ edição, São Paulo, 2000. Edit by Manole.

33. Stinear, C., Barber, L., Smale, P., Coxon, J., Fleming, M., Byblow, W. Functional potential in chronic stroke patients depends on corticospinal tract integrity. Brain (2007), 130, 170-180

34. Teixeira-S., Olney J., Nadeau S, Brouwer B. Muscle strengthening and physical conditioning to reduce impairment and disability in chronic stroke survivors. Arch Phys Med Rehabil. 1999. 80:1211-1218.

35. Van S. Classificatie van links - en rechtshangige proefperrsonen. Nederlands Tijdsschrift voor de Psycologie. 2002. 47:88-92.

36. Winstein, C., Rose, D., Tan, S., Lewthwaite, R., Chui, H.C. A randomized controlled comparison of upper extremity rehabilitation in acute stroke: immediate and longer-term outcomes. Archives of physical Medicine and Rehabiliattion. 2004. 85: 620-628.

37. Wang, R., Chan R., Tsai M., Effects of thoraco lumbar electric sensory stimulation on knee extensor spasticity of persons who survived cerebrovascular accident (CVA). Journal of Rehabilitation Research and Development. 2000. $37.73-80$.

38. Whitall, J., Waller, S., Silver, K., Macko, F. Repetitive bilateral arm training with rhythmic auditory cueing improves motor function in chronic hemiparetic stroke. American Heart Association. 2000. 31:2390 